\title{
Current status of lymph node micrometastasis in gastric cancer
}

\author{
Yang Zhou ${ }^{1}$, Guo-Jing Zhang ${ }^{1}$, Ji Wang ${ }^{1}$, Kai-Yuan Zheng ${ }^{1}$ and Weihua Fu ${ }^{1}$ \\ 1 Tianjin Medical University General Hospital, Tianjin, China \\ Correspondence to: Weihua Fu, email: tjmughgs_fwh@163.com \\ Keywords: gastric cancer; lymph nodes metastasis; micrometastasis; molecular technique; minimally invasive surgery \\ Received: December 08, $2016 \quad$ Accepted: April 03, $2017 \quad$ Published: April 27, 2017
}

Copyright: Zhou et al. This is an open-access article distributed under the terms of the Creative Commons Attribution License 3.0 (CC BY 3.0), which permits unrestricted use, distribution, and reproduction in any medium, provided the original author and source are credited.

\section{ABSTRACT}

Lymph node metastasis is one of the most important prognostic factors in patients with gastric cancer. An inadequate number of dissected lymph nodes is an independent risk factor affecting recurrence, even in patients who are node negative. Oddly, certain early-stage patients still experience recurrence or metastasis within a short time, even if they have undergone standard radical mastectomy. Many researchers have attributed these adverse events to lymph node micrometastasis (LNM), which is defined as a microscopic deposit of malignant cells of less than $\mathbf{2} \mathbf{~ m m}$ in diameter. With the development of diagnostic tools such as immunohistochemistry and reverse transcription-polymerase chain reaction, the rate of detection of LNM has been constantly increasing. Although there is no clear consensus about risk factors for or the definitive clinical significance of LNM, the clinical impact of LNM is remarkable in gastric cancer. For minimally invasive treatment in particular, such as endoscopic submucosal dissection and laparoscopic surgery, accurate diagnosis of LNM is regarded as the potential key to maintaining the balance between curability and safety. This review provides an overview of the definition, detection and significance of LNM in gastric cancer. We also summarize several attention-drawing controversies regarding the treatment of patients who may have LNM.

\section{INTRODUCTION}

Gastric cancer remains one of the most frequently occurring malignancies. It is the third leading cause of cancer-related deaths worldwide, and its prevalence is increasing [1]. Generally, patients with node-negative gastric carcinoma have a good prognosis compared with patients who are node positive [2]. In China, the many new patients who are diagnosed at advanced stages have missed the best opportunity for curative surgery [3]. Even for patients without node metastasis, there is a certain recurrence rate after extensive lymphadenectomy [4]. Historically, representative sections from removed nodes are used in histological examination, and lymph node micrometastasis (LNM) that is not detected by routine pathologic examination may be identified in multiple sections of lymph nodes. The detection of LNM has been exposed as the weakness of histological examination, and this has prompted researchers to seek a more sensitive way to characterize micrometastasisrelated tumour cells. Due to technological developments, such as immunohistochemistry (IHC) and reverse transcription-polymerase chain reaction (RT-PCR), the rate of diagnosis of micrometastasis has increased significantly. Comparatively speaking, IHC can offer satisfactory accuracy for the detection of LNM, whereas RT-PCR is more sensitive but may offer falsepositive results caused by various sources of biological contamination. LNM has increasingly been the focus of research based on these diagnostic tools. However, there have been many controversies about the impact of LNM, such that no consensus on the clinical treatment or significance of micrometastatic node involvement in patients with gastric cancer has been reached. In recent years, with the rise of endoscopic submucosal dissection (ESD), laparoscopic surgery and other minimally invasive treatments have been widely performed in consideration of postsurgical quality of life (QOL). However, LNM may have an unfavourable influence on recurrence after these unconventional treatments, and the need to balance the relationship between QOL and safety is making research on micrometastasis more urgent.

\section{DEFINITION OF MICROMETASTASIS}

LNM was initially defined as a microscopic deposit of malignant cells of less than $2 \mathrm{~mm}$ in diameter [5]. This deposit has the ability to escape immune supervision 
and to progress to macroscopic malignant growth. With deeper study, the Union for International Cancer Control (UICC) redefined single tumour cells or cell clusters measuring $\leq 0.2 \mathrm{~mm}$ in the greatest dimension as "isolated tumour cells" (ITCs) because of their different biological behaviour and size. In other words, micrometastasis was defined as referring to tumour cell clusters between 0.2 $\mathrm{mm}$ and $2 \mathrm{~mm}$ in the greatest dimension [6]. The effort was aimed at accurate staging and distinguishing the entities' different biological behaviours. According to the 7th TNM classification by the UICC, first, LNM should be considered in node staging of gastric cancer [7]. Patients with LNM are staged as pN1(mi), and patients with ITCs in the lymph nodes are staged as pNO(i+). Moreover, if micrometastasis can be detected only by RT-PCR, its N stage should be determined as $\mathrm{pN} 0(\mathrm{~mol}+)$ [8]. Second, ITCs do not show evidence of metastatic activity, such as proliferation or penetration of the vascular system, compared with LNM. LNM is not equal to lymph node metastasis mainly because of different outcomes. The lack of neovascularization means that tumour sizes cannot exceed $3 \mathrm{~mm}$. At the same time, the cells may be in a state between proliferation and death. VEGF-C is considered the key factor in breaking dormancy [9]. The uncertain mechanisms of and conditions for LNM growth are responsible for the very different conclusions reported from different centres.

\section{DETECTION OF MICROMETASTASIS}

\section{Serial sectioning}

Serial sectioning has been applied in several methods for LNM detection, and subsequently, the rate of detection of nodal micrometastasis markedly increased compared with that for HE staining, which is limited because many small tumour cell clusters may be overlooked [10]. Approximately $40 \%$ of patients classified as pNO according to traditional HE staining methods have nodal micrometastasis. With narrower slice intervals, the detection rate gradually increases. However, the weaknesses of a heavy workload and low efficiency have limited this approach's application, so it has been supplemented with or replaced by more sensitive procedures.

\section{Immunohistochemical staining}

Small lesions not visible by routine pathologic examination can be detected by IHC, which has been utilized as a standard tool for detecting LNM in gastric cancer. Many studies have confirmed significantly higher sensitivity for immunohistochemical methods than for HE staining. Meanwhile, cytokeratin (CK) AE1/AE3 and CAM5.2 have been identified as reliable markers of epithelial cells in lymph nodes [11]. These markers' biggest advantage is their ability to distinguish a single tumour cell from small clusters of tumour cells using morphological distinctions, and this technique has been shown to be invaluable in retrospective studies. In 2003, Matsumoto et al. introduced a rapid IHC technique to diagnose LNM within 30 minutes during operations for gastric cancer [12]. The incidence of LNM according to different reports is diverse. For T1-stage cases, Maehara et al. [13] and Kashimura et al. [14] reported that the incidence of LNM was $23.5 \%$ and $23.4 \%$, respectively. However, Kim et al. [15] reported that the incidence was only $10 \%$ in pT1N0 patients. For T2-stage cases, according to a report by Kikuchi et al., the rate was $43.2 \%$ [16]. Considering these research results, the incidence of LNM seems to be related to deep tumour invasion. The lack of consensus on this topic is a problem, and more experimentation is needed to evaluate the issue. The number of lymph node sections required is another issue in the application of IHC. According to a study that included 98 patients with colorectal cancer, Noura et al. [17] argued that at least five sections should be assessed. There have been no related studies on gastric cancer. Compared with RT-PCR, the advantages of immunohistochemical methods are their availability, their intuitive results and their lower costs, but these methods are more likely to be affected by the experimental techniques and conditions used, so they tend to be less accurate [18].

\section{RT-PCR assay}

The greatest advantage of RT-PCR is its sensitivity, such that 1 tumour cell in $1 \times 10^{7}$ cells can be detected [19]. The introduction of RT-PCR increased the rate of detection of LNM to a level higher than that achieved using IHC [20]. The amplification of gene transcripts associated with occult tumour cells is a reason for the higher sensitivity of RT-PCR. The key in using the RT-PCR method is to select an appropriate target marker. Tissue-specific genes, such as CEA, tend to be used [20] because no gastrointestinal cancer-specific marker genes have been discovered and because CEA is expressed in most cancers and in normal gastrointestinal cells. Generally, the rate of detection of LNM is greater than 30\%. Jagric et al. [21] published a study in which micrometastasis was detected in $40 \%$ of patients. History justifies our confidence in the method but also shows that it is not fool proof. Marker-related pseudogenes, incorrect transcription of genes and various sources of biological contamination are responsible for false positives. In contrast, heterogeneous expression of a target marker can lead to false negatives. Although RTPCR sometimes offers false results, this technique is still considered to be the most accurate option for the detection of LNM. Kubota et al. [22] reported that the detection rates of RT-PCR and IHC were $9.9 \%$ and $3.6 \%$, respectively. 
Table1: IHC and RT-PCRI studies in gastric cancer patients with node negativity diagnosed by HE staining

\begin{tabular}{|c|c|c|c|c|c|c|}
\hline Year & Study & No.of patients & \begin{tabular}{|l|l|} 
Pathologic results by \\
HE
\end{tabular} & Methods & Target markers & Incidence(\%) \\
\hline 1996 & Maehara et al.[13] & 34 & pT1N0 & $\mathrm{IHC}$ & CK(CAM5.2) & $23.5 \%$ \\
\hline 1999 & Kashimura et al.[14] & 47 & pT1bN0 & IHC & CK(CAM5.2) & $23.4 \%$ \\
\hline 2000 & Harrison et al.[44] & 25 & pT1-4N0 & $\mathrm{IHC}$ & CK(CAM5.2) & $36.0 \%$ \\
\hline 2000 & Cai et al.[45] & 69 & pT1bN0 & $\mathrm{IHC}$ & CK(CAM5.2) & $24.6 \%$ \\
\hline 2001 & Fukagawa et al.[34] & 107 & pT2-3N0 & $\mathrm{IHC}$ & $\mathrm{CK}(\mathrm{AE} 1 / \mathrm{AE} 3)$ & $35.5 \%$ \\
\hline 2001 & Morgagni et al.[46] & 139 & pT1N0 & $\mathrm{IHC}$ & CK(MNF 116) & $17.3 \%$ \\
\hline 2001 & Nakajo et al.[27] & 67 & pT1-3N0 & $\mathrm{IHC}$ & $\mathrm{CK}(\mathrm{AE} 1 / \mathrm{AE} 3)$ & $14.9 \%$ \\
\hline 2002 & Lee at al.[47] & 41 & pT1N0 & $\mathrm{IHC}$ & CK(AE1/AE3) & $24.4 \%$ \\
\hline 2002 & Yasuda et al.[30] & 64 & pT2-4aN0 & $\mathrm{IHC}$ & CK(CAM5.2) & $31.3 \%$ \\
\hline 2002 & Choi et al.[48] & 88 & pT1bN0 & $\mathrm{IHC}$ & $\mathrm{CK}(35 \beta \mathrm{H} 11)$ & $31.8 \%$ \\
\hline 2003 & Morgagni et al.[28] & 300 & pT1N0 & $\mathrm{IHC}$ & CK(MNF 116) & $10.0 \%$ \\
\hline 2006 & Miyake et al.[49] & 120 & pT1N0 & IHC & CK(AE1/AE3) & $22.5 \%$ \\
\hline 2007 & Yonemura et al.[50] & 308 & pT1-4N0 & IHC & CK(AE1/AE3) & $12 \%$ \\
\hline 2008 & Kim et al.[51] & 184 & pT1-4aN0 & $\mathrm{IHC}$ & CK(AE1/AE3) & $16.8 \%$ \\
\hline 2008 & Ishii et al.[52] & 35 & pT1b-2N0 & $\mathrm{IHC}$ & CK(O.N>352) & $11 \%$ \\
\hline 2011 & Cao et al.[32] & 160 & pT1N0 & $\mathrm{IHC}$ & CK(AE1/AE3) & $21.3 \%$ \\
\hline 2011 & Wang et al.[53] & 191 & pT1-3N0 & $\mathrm{IHC}$ & $\mathrm{CK}(\mathrm{AE} 1 / \mathrm{AE} 3)$ & $28.3 \%$ \\
\hline 2012 & Ru et al.[29] & 45 & pT1-4N0 & $\mathrm{IHC}$ & CK(19),CD44v6 & $33.3 \%$ \\
\hline 2001 & Okada et al.[54] & 24 & \begin{tabular}{|l|} 
pT1-4aN0 \\
\end{tabular} & RT-PCR & EA,CK20, & $41.7 \%$ \\
\hline 2002 & Matsumoto et al.[20] & 50 & \begin{tabular}{|l|} 
pT1-4N0 \\
\end{tabular} & RT-PCR & CEA & $28 \%$ \\
\hline 2005 & Arigami et al.[55] & 80 & pT1-3N0 & RT-PCR & CEA & $31.3 \%$ \\
\hline 2006 & Sonoda et al.[56] & 33 & pT1N0 & RT-PCR & MUC 2,TFF 1 & $33.3 \%$ \\
\hline 2007 & Wu et al.[57] & 10 & - & RT-PCR & CK20 & $20 \%$ \\
\hline
\end{tabular}

Similarly, the respective outcomes reported by Arigami et al. were $31.3 \%$ and $11.3 \%$. To avoid false detection results, a multiplex RT-PCR assay has been recommended for application [23]. In the future, this molecular system is expected to become an intraoperative diagnostic tool for the rapid detection of LNM. For this reason, the system may be regarded as a protective measure for patients undergoing minimally invasive surgery with personalized lymphadenectomy (Table 1).

\section{CLINICAL SIGNIFICANCE OF NODAL MICROMETASTASIS}

\section{LNM and the staging system}

The staging of gastric cancer is an important and controversial issue. The significance of micrometastasis is often obscured by macrometastasis. Many researchers have focused on the detection of LNM in patients with pN0-stage cancer and regard it as an early event indicating the possibility of clinical metastasis. In the past, researchers attached importance to macrometastasis and disregarded micrometastasis. However, chronic negligence of LNM may have led to underestimation of the disease state. According to the 7th edition of the
American Joint Committee on Cancer system [24], the $\mathrm{N}$ stage is determined by the number of metastatic nodes [24]. Furthermore, when the number of metastatic nodes exceeds 15 , the detection of LNM is unnecessary because the situation has already indicated the most advanced $\mathrm{N}$ stage. However, the detection of LNM could improve staging when the number of metastatic nodes is less than 15 . In other words, the $\mathrm{N}$ stage may vary with the detection of LNM [25]. Lee et al. [26] reported that the rate of detection of LNM was $32.4 \%$ in 482 patients and that the patients with LNM had a worse 5-year survival rate. The authors surmised that ignorance of LNM, which may have the same prognostic value as lymph node macrometastasis, causes the tumour stage to be underestimated, resulting in an overly optimistic conclusion regarding the disease state. Many institutes have not recognized the value of LNM in the staging process. Accounting for this problem, Lee et al. [26] recommended a new staging system that included LNM. Compared with conventional staging systems, the researchers found that in their staging system, the prognostic difference between stages N2 and N3a was more distinct. This exciting result implied that LNM could affect staging in patients with advanced gastric cancer as well as in patients with early gastric cancer. 


\section{The prognostic value of LNM}

Results for the predictive value of LNM have varied between different studies [27, 28]. Many researchers have suggested that patients with LNM have a poor prognosis $[29,30]$. The prognosis of gastric cancer is relevant to the tumour stage, and the discovery of LNM often means that the stage of the tumour improved spontaneously but that the prognosis is poor [31]. Using gastric cancer-related death as the end point, Li et al. [31] found that the 5-year survival rates of patients with LNM were significantly poorer than those of patients without LNM $(\mathrm{HR}=2.81$, 95\% CI 1.96 4.02). Lee et al. [26] and Cao et al. [32] came to the same conclusion. In the first meta-analysis of the influence of LNM on the prognosis of gastric cancer, the researchers declared that in patients with gastric carcinoma, LNM was associated with a higher recurrence rate [33], but LNM should not be seen as the gold standard for prognosis evaluation in patients with gastric cancer. Conversely, certain researchers have suggested that LNM does not affect the prognosis of patients. A study from the National Cancer Center Hospital in Tokyo showed that the presence of nodal micrometastasis does not correlate with survival in patients with advanced gastric cancer. A study including 300 patients with stage $\mathrm{T}_{1} \mathrm{~N}_{0}$ cancer also showed that LNM did not affect the survival rate of patients at 5 years or 10 years [28]. The host immune response was considered to be one of the reasons for this phenomenon. Another study comprising 107 patients with $\mathrm{T}_{2} \mathrm{~N}_{0} \mathrm{M}_{0}$ cancer also showed that the 5-year survival rates of patients with or without LNM were $89 \%$ and $94 \%$, respectively; the difference was not statistically significant [34]. The prognostic value of LNM remains controversial, so an increasing number of researchers have aimed to explain the reasons for this variation by exploring the conditions required for micrometastasis progression to macroscopic malignant growth. Our knowledge of the natural growth history is increasing but remains limited. We believe that the heterogeneity of LNM among patients is responsible for the controversy. The sources of heterogeneity may include different approaches to staging, different therapeutic regimens, and different physical states, among others. The effects of these confounding factors on LNM make it difficult to identify the most important factor. In particular, if the effects of these differences could be eliminated, studies might be able to reach a relatively reliable conclusion.

\section{CLINICAL TREATMENT STRATEGIES}

\section{Traditional surgery}

Japanese researchers have emphasized the significance of D2 lymphadenectomy for patients with gastric carcinoma, whereas Western researchers have suggested that it does not show a survival advantage compared with D1 radical surgery [35]. In Asia in particular, a large number of studies have confirmed the superiority of a D2 operation, which is considered the standard surgical procedure for gastric cancer [36]. The phenomena of micrometastasis and jumping metastases also seem to suggest that reduction of the scope of the operation should be performed with caution. As previously described, the incidence of LNM in gastric cancer is higher than $10 \%$, even in both mucosal and submucosal cancers. As a potential clinical metastasis source, LNM can become a source of recurrence once micrometastasis is activated. The existence of LNM also increases the rationality of the expansion of lymph node dissection, or at least the scope of the operation should not be easily reduced, especially in the diffuse type of gastric cancer. A number of researchers have declared that extensive lymphadenectomy can improve the survival rate of gastric cancer patients without lymph node metastasis, which may be related to the reduction of the presence of LNM. Meanwhile, many researchers have argued that LNM is not equivalent to clinical metastasis and may be cleared depending on the defence system and immune system capabilities. Even if the first line of natural defence is broken, LNM may still be effectively inactivated with the help of preoperative or postoperative adjuvant chemotherapy. Certain radical researchers have even suggested that extensive lymphadenectomy is only beneficial to improve the accuracy of staging [37].

\section{Endoscopic resection}

In recent years, ESD has been increasingly suggested to replace radical resection in the treatment of early gastric cancer because of its better postoperative QOL [38]. The implementation of ESD must meet two conditions: (1)there is no lymph node metastasis, and (2)the disease focus can be excised all at once. The evaluation of lymph node status is the most important precondition [39]. An accurate intraoperative evaluation is necessary when performing minimally invasive surgery because the clinical impact of metastasis is contentious, i.e., ESD can be applied when lymph node metastasis is not expected [40]. Chinese researchers have found that the tumour invasion depth, lymph node metastasis and vascular invasion may affect the prognosis of patients with early gastric cancer but that only lymph node metastasis is an independent prognostic factor. If the treatment can be performed based on accurate assessment of lymph node status, the relationship between postoperative QOL and the safety of treatment can be well balanced. However, Japan's Guide for the Treatment of Gastric Cancer (April 2010 Edition) [41] continues to retain the expanded ESD/ endoscopic mucosal resection (EMR) indications. At the same time, the guide emphasizes that more clinical trials 
are needed due to the lack of sufficient clinical evidence. If we consider the concept of LNM, these terms cause more concerns regarding the techniques' application. For all diagnostic tools, none had an accuracy greater than $90 \%$, and pathologic examination remains the gold standard for assessing lymph node metastases. Thus, EMR/ESD bears the risk of missing lymph node metastases. The results of research on the accuracy of ESD from Japan and South Korea were significantly more optimistic than those from Europe and the United States; this may be related to differences in the ESD pointer, the technique and other aspects. Japan's National Cancer Center conducted a massive study in which 1,955 people with early gastric cancer were included, and it was found that the 5-year survival rates for ESD and radical mastectomy were not significantly different [42]. Another large sample study including 1,485 patients with early-stage cancer showed that the 5-year survival rate for ESD was $92.4 \%$. There was no statistically significant difference between ESD and radical mastectomy operations. In addition, certain Western studies have shown that the safety of ESD is relatively poor. In contrast, the recurrence rate is significantly lower in patients with early gastric cancer without lymph node metastasis who underwent standard radical mastectomy. With progress in detection, the rate of detection of LNM after operation has greatly improved. In addition, early gastric cancer has its own special metastatic form and mechanism [43]. Therefore, considering the concept of LNM and endoscopic treatment, performing local resection of tumours instead of expanding lymph node dissections is controversial. The risk of ESD/EMR should also be re-evaluated. To answer these questions, a large number of studies on the mechanisms of and conditions for LNM growth are also required. Because of issues with operating techniques and security, among other issues, ESD technology has not yet been promoted in Europe or the United States.

\section{CONCLUSIONS}

Nearly all the recent studies on lymph node metastasis have been retrospective analyses. There is a lack of studies on the mechanisms of LNM proliferation. This is precisely the key to breaking through the bottleneck in current research. With in-depth study of its biological behaviour, we believe that there will be a more reasonable and individualized comprehensive treatment plan for patients with gastric cancer, and especially patients with early gastric cancer. Looking forward, minimally invasive surgery may be safely performed and may achieve satisfactory results with regard to the balance between QOL and curability.

\section{CONFLICTS OF INTEREST}

The authors declare no conflict of interest.

\section{REFERENCES}

1. Ferlay J, Soerjomataram I, Ervik M, Dikshit R, Eser S, Mathers C, Rebelo M, Parkin DM, Forman D, Bray F, Editors. Globocan 2012: Estimated cancer incidence, mortality and prevalence worldwide in 2012. IARC Cancer Database; 2012.

2. Jeuck TL, Wittekind C. Gastric carcinoma: stage migration by immunohistochemically detected lymph node micrometastases. Gastric Cancer. 2015; 18: 100-8.

3. Lin Y, Ueda J, Kikuchi S, Totsuka Y, Wei WQ, Qiao YL, Inoue M. Comparative epidemiology of gastric cancer between Japan and China. World J Gastroenterol. 2011; 17: 4421-8.

4. Zhou Y, Yu F, Wu L, Ye F, Zhang L, Li Y. Survival after Gastrectomy in Node-Negative Gastric Cancer: A Review and Meta-Analysis of Prognostic Factors. Medical science monitor. 2015; 21: 1911.

5. Sobin LH, Gospodarowicz MK, Wittekind C. International Union against cancer. TNM classification of malignant tumours. Chichester, West Sussex, UK; Hoboken. NJ: Wiley-Blackwell; 2010.

6. Lee CM, Park SS, Kim JH. Current Status and Scope of Lymph Node Micrometastasis in Gastric Cancer. Journal of gastric cancer. 2015; 15: 1-9.

7. Yoon H, Lee DH. New approaches to gastric cancer staging: beyond endoscopic ultrasound, computed tomography and positron emission tomography. World J Gastroenterol. 2014; 20: 13783-90.

8. Fukagawa T, Sasako M, Ito S, Nakanishi H, Iinuma $H$, Natsugoe S, Katai H, Shimoda T. The prognostic significance of isolated tumor cells in the lymph nodes of gastric cancer patients. Gastric Cancer. 2010; 13: 191-6.

9. Pérez D, Rohde A, Callejón G, Pérez-Ruiz E, Rodrigo I, Rivas-Ruiz F, Ramos B, Medina F, Villatoro R, Redondo $\mathrm{M}$. Correlation between serum levels of vascular endothelial growth factor-C and sentinel lymph node status in early breast cancer. Tumor Biology. 2015; 36: 9285-93.

10. Isozaki H, Okajima K, Fujii K. Histological evaluation of lymph node metastasis on serial sectioning in gastric cancer with radical lymphadenectomy. Hepato-gastroenterology. 1996; 44: 1133-6.

11. Hata M, Machi J, Mamou J, Yanagihara ET, SaegusaBeecroft E, Kobayashi GK, Wong CC, Fung C, Feleppa EJ, Sakamoto K. Entire-volume serial histological examination for detection of micrometastases in lymph nodes of colorectal cancers. Pathology \& Oncology Research. 2011; 17: 835-41.

12. Matsumoto $\mathrm{M}$, Natsugoe $\mathrm{S}$, Ishigami S, Uenosono $\mathrm{Y}$, Takao S, Aikou T. Rapid immunohistochemical detection of lymph node micrometastasis during operation for upper gastrointestinal carcinoma. British journal of surgery. 2003; 90: 563-6.

13. Maehara Y, Oshiro T, Endo K, Baba H, Oda S, Ichiyoshi 
Y, Kohnoe S, Sugimachi K. Clinical significance of occult micrometastasis in lymph nodes from patients with early gastric cancer who died of recurrence. Surgery. 1996; 119: 397-402.

14. Kashimura H, Ajioka Y, Watanabe H, Nishikura K, Iiri T, Asakura H. Risk factors for nodal micrometastasis of submucosal gastric carcinoma: assessment of indications for endoscopic treatment. Gastric Cancer. 1999; 2: 33-9.

15. Kim JJ, Song KY, Hur H, Hur JI, Park SM, Park CH. Lymph node micrometastasis in node negative early gastric cancer. Eur J Surg Oncol. 2009; 35: 409-14.

16. Kikuchi Y, Tsuchiya A, Ando Y, Yoshida T, Takenosita S. Immunohistochemical detection of lymph node microinvolvement in node-negative gastric cancer. Gastric Cancer. 1999; 2: 173-8.

17. Noura S, Yamamoto H, Miyake Y, Kim Bn, Takayama O, Seshimo I, Ikenaga M, Ikeda M, Sekimoto M, Matsuura $\mathrm{N}$, Monden M. Immunohistochemical assessment of localization and frequency of micrometastases in lymph nodes of colorectal cancer. Clinical cancer research. 2002; 8: 759-67.

18. Torlakovic EE, Nielsen S, Vyberg M, Taylor CR. Getting controls under control: the time is now for immunohistochemistry. Journal of clinical pathology. 2015; 68:879-82.

19. Muto Y, Matubara H, Tanizawa T, Nabeya Y, Kawahira H, Akai T, Hoshino I, Hayashi H. Rapid diagnosis of micrometastasis of gastric cancer using reverse transcription loop-mediated isothermal amplification. Oncology reports. 2011; 26: 789-94.

20. Matsumoto M, Natsugoe S, Ishigami S, Nakashima S, Nakajo A, Miyazono F, Hokita S, Takao S, Eizuru Y, Aikou T. Lymph node micrometastasis and lymphatic mapping determined by reverse transcriptase-polymerase chain reaction in pN0 gastric carcinoma. Surgery. 2002; 131: 6305.

21. Jagric T, Ivanecz A, Horvat M, Plankl M, Kavalar R, Kavalar S, Mis K, Mars T. Evaluation of a focused sentinel lymph node protocol in node-negative gastric cancer patients. Hepato-gastroenterology. 2012; 60: 1231-6.

22. Kubota K, Nakanishi H, Hiki N, Shimizu N, Tsuji E, Yamaguchi H, Mafune Ki, Tange T, Tatematsu M, Kaminishi M. Quantitative detection of micrometastases in the lymph nodes of gastric cancer patients with real-time RT-PCR: A comparative study with immunohistochemistry. International journal of cancer. 2003; 105: 136-43.

23. Kuo CT, Hoon DS, Takeuchi H, Turner R, Wang HJ, Morton DL, Taback B. Prediction of disease outcome in melanoma patients by molecular analysis of paraffinembedded sentinel lymph nodes. Journal of clinical oncology. 2003; 21: 3566-72.

24. Washington K. 7th edition of the AJCC cancer staging manual: stomach. Annals of surgical oncology. 2010; 17: 3077-9.
25. Kim JH, Park SS, Park SH, Kim SJ, Mok YJ, Kim CS, Lee JH, Kim YS. Clinical significance of immunohistochemically-identified lymphatic and/or blood vessel tumor invasion in gastric cancer. Journal of Surgical Research. 2010; 162: 177-83.

26. Lee CM, Cho JM, Jang YJ, Park SS, Park SH, Kim SJ, Mok YJ, Kim CS, Kim JH. Should Lymph Node Micrometastasis be Considered in Node Staging For Gastric Cancer? Annals of surgical oncology. 2015; 22: 765-71.

27. Nakajo A, Natsugoe S, Ishigami S, Matsumoto $M$, Nakashima S, Hokita S, Baba M, Takao S, Aikou T. Detection and prediction of micrometastasis in the lymph nodes of patients with pN0 gastric cancer. Annals of surgical oncology. 2001; 8: 158-62.

28. Morgagni P, Saragoni L, Scarpi E, Zattini PS, Zaccaroni A, Morgagni D, Bazzocchi F. Lymph node micrometastases in early gastric cancer and their impact on prognosis. World journal of surgery. 2003; 27: 558-61.

29. Ru Y, Zhang L, Chen Q, Gao S, Wang G, Qu Z, Shan T, Qian N, Feng X. Detection and clinical significance of lymph node micrometastasis in gastric cardia adenocarcinoma. Journal of International Medical Research. 2012; 40: 293-9.

30. Yasuda K, Adachi Y, Shiraishi N, Inomata M, Takeuchi H, Kitano S. Prognostic effect of lymph node micrometastasis in patients with histologically node-negative gastric cancer. Annals of surgical oncology. 2002; 9: 771-4.

31. Lee T, Tanaka H, Ohira M, Okita Y, Yoshii M, Sakurai K, Toyokawa T, Kubo N, Muguruma K, Tanaka S. Clinical impact of the extent of lymph node micrometastasis in undifferentiated-type early gastric cancer. Oncology. 2014; 86: 244-52.

32. Cao L, Hu X, Zhang Y, Huang G. Adverse prognosis of clustered-cell versus single-cell micrometastases in pN0 early gastric cancer. Journal of surgical oncology. 2011; 103: 53-6.

33. Zeng YJ, Zhang CD, Dai DQ. Impact of lymph node micrometastasis on gastric carcinoma prognosis: A metaanalysis. World journal of gastroenterology. 2015; 21: 1628.

34. Fukagawa $T$, Sasako $M$, Mann GB, Sano $T$, Katai H, Maruyama K, Nakanishi Y, Shimoda T. Immunohistochemically detected micrometastases of the lymph nodes in patients with gastric carcinoma. Cancer. 2001; 92: 753-60.

35. Degiuli M, Sasako M, Ponti A, Vendrame A, Tomatis M, Mazza C, Borasi A, Capussotti L, Fronda G, Morino M. Randomized clinical trial comparing survival after D1 or D2 gastrectomy for gastric cancer. British Journal of Surgery. 2014; 101: 23-31.

36. Chen XZ, Hu JK, Zhou ZG, Rui YY, Yang K, Wang L, Zhang B, Chen ZX, Chen JP. Meta-analysis of effectiveness and safety of D2 plus para-aortic lymphadenectomy for resectable gastric cancer. Journal of the American College 
of Surgeons. 2010; 210: 100-5.

37. Bonenkamp J, Van De Velde C, Hermans J, Siewert J, Roder J. Randomised trial of extended lymph node dissection for gastric cancer. Proceedings of the 2nd International Gastric Cancer Congress, Munich; 1997. pp. 1111-6.

38. Gotoda T. Endoscopic resection of early gastric cancer. Gastric cancer. 2007; 10: 1-11.

39. Kume K. Endoscopic therapy for early gastric cancer: standard techniques and recent advances in ESD. World journal of gastroenterology. 2014; 20: 6425.

40. Jee Y, Hwang SH, Rao J, Park D, Kim HH, Lee HJ, Yang HK, Lee K. Safety of extended endoscopic mucosal resection and endoscopic submucosal dissection following the Japanese Gastric Cancer Association treatment guidelines. British Journal of Surgery. 2009; 96: 1157-61.

41. Association JGC. Japanese classification of gastric carcinoma: 3rd English edition. Gastric cancer. 2011; 14: 101-12.

42. Gotoda T, Iwasaki M, Kusano C, Seewald S, Oda I. Endoscopic resection of early gastric cancer treated by guideline and expanded National Cancer Centre criteria. British Journal of Surgery. 2010; 97: 868-71.

43. Gurzu S, Jung I, Kadar Z. Aberrant metastatic behavior and particular features of early gastric cancer. Apmis. 2015; 123: 999-1006. 